\title{
IMU Dataset For Motion and Device Mode Classification
}

Parinaz Kasebzadeh, Gustaf Hendeby, Carsten Fritsche, Fredrik Gunnarsson and Fredrik Gustafsson

The self-archived postprint version of this journal article is available at Linköping University Institutional Repository (DiVA):

http:/ / urn.kb.se/ resolve?urn=urn:nbn:se:liu:diva- 143958

N.B.: When citing this work, cite the original publication.

Kasebzadeh, P., Hendeby, G., Fritsche, C., Gunnarsson, F., Gustafsson, F., (2017), IMU Dataset For Motion and Device Mode Classification, 2017 INTERNATIONAL CONFERENCE ON INDOOR POSITIONING AND INDOOR NAVIGATION (IPIN). https:// doi.org/ 10.1109/ IPIN.2017.8115956

Original publication available at:

https:/ / doi.org/ 10.1109/IPIN.2017.8115956

Copyright: IEEE

http:// www.ieee.org/

(C) 2017 IEEE. Personal use of this material is permitted. However, permission to reprint/republish this material for advertising or promotional purposes or for creating new collective works for resale or redistribution to servers or lists, or to reuse any copyrighted component of this work in other works must be obtained from the IEEE. 


\title{
IMU Dataset For Motion and Device Mode Classification
}

\author{
Parinaz Kasebzadeh, Gustaf Hendeby, Carsten Fritsche, Fredrik Gunnarsson ${ }^{\dagger}$, Fredrik Gustafsson \\ Department of Electrical Engineering, Linköping University, Linköping, Sweden \\ Email: \{firstname.lastname\}@liu.se \\ † Ericsson Research, Linköping, Sweden, Email: fredrik.gunnarsson@ericsson.com
}

\begin{abstract}
Classification of motion mode (walking, running, standing still) and device mode (hand-held, in pocket, in backpack) is an enabler in personal navigation systems for the purpose of saving energy and design parameter settings and also for its own sake. Our main contribution is to publish one of the most extensive datasets for this problem, including inertial data from eight users, each one performing three pre-defined trajectories carrying four smartphones and seventeen inertial measurement units on the body. All kind of metadata is available such as the ground truth of all modes and position. A second contribution is the first study on a joint classifier of motion and device mode, respectively, where preliminary but promising results are presented.
\end{abstract}

\section{INTRODUCTION}

Pedestrian navigation systems (PNS) are used in a range of applications, from pure navigation and guidance tools, healthcare assistance systems to infotainment applications and more generally location based services. The main goal of a PNS is to have an accurate and reliable position estimate, but there are certain metadata that can provide additional information in itself. In this contribution, the problem of classifying the activity mode (standing still, walking, running) and the device mode (handheld in view, handheld in swinging hand, in front/back pocket, and in a backpack) is considered.

Our study is based on an extensive experimental study where different users repeated the same trajectories and sequences of modes. We logged data from low cost microelectromechanical systems (MEMS) sensors including acceleration, angular rate, magnetic field, barometric pressure and also GPS as a position reference. Data were measured by using four smartphones and 17 inertial measurement units (IMUs) configured in a body suit. The IMUs generate data of somewhat higher accuracy than the smartphones, and the body suit software makes use of advanced biomechanical models to provide accurate description of true motion of all body parts, which in turn could be used to simulate data from any other part of the body. Ground truth of the device and motion mode is available from the experimental setup. Table I summarizes different device and motion modes. In order to simplify referring to each of these scenarios, Table I also assigns a specific class to each of them. We believe this to be one of the most extensive datasets publicly available for PNS.

978-1-5090-6299-7/17\$31.00 (c) 2017 IEEE
TABLE I: Motion-Device mode Classification

\begin{tabular}{|c|c|c|c|}
\hline $\begin{array}{lc}\text { Motion } \\
\text { Mode }\end{array}$ & $\begin{array}{l}\text { Standing Still } \\
\text { (SS) }\end{array}$ & $\begin{array}{l}\text { Walking } \\
\text { (W) }\end{array}$ & $\begin{array}{c}\text { Running } \\
\text { (R) }\end{array}$ \\
\hline Fixed hand (1) & \multirow{4}{*}{ Class SS } & Class W1 & Class R1 \\
\hline Swinging hand (2) & & Class W2 & Class R2 \\
\hline Pocket (3) & & Class W3 & Class R3 \\
\hline Backpack (4) & & Class W4 & Class R4 \\
\hline
\end{tabular}

The activity mode is a key feature in sports and healthcare applications, where it is logged for its own sake. The activity mode can also select a set of appropriate internal parameters in the PNS, such as step length and step detection thresholds [1]. It can also be an enabler for energy efficient PNS. For instance, in outdoor applications, an energy demanding GPS fix can be obtained first when the user has moved a certain distance, and here it is useful to know if the user is standing still, walking or running. Similar compromises about using additional information sources from infrastructure can be made in indoor PNS. For certain personnel such as guards and rangers, running may indicate danger and a sudden and unexpected stand still can indicate an accident, and in both cases officers can be automatically alerted.

The device mode is crucial for the design and performance of a PNS. For instance, if it is known that the device is rigidly attached to a foot, special tricks can be used [2]-[4]. Most importantly, using the knowledge that the foot is at rest at least for a short while in each stance, the bias in the accelerometer and gyroscope can be read off directly, This is referred to as zero velocity updates (ZUPTs) and zero angular rate updates (ZARUs), respectively. The elimination of bias enables the use of dead-reckoning principles to integrate acceleration and angular rate into a precise trajectory.

Other assumptions on the device mode include that the IMU is fixed on the waist rather than the foot [5], [6], located in the front pocket [7], carried horizontally in hand [8] or carried in hand not necessarily horizontally [9]-[12].

Classification of various motion modes could be one step towards more realistic scenarios in which the smartphone is allowed to switch arbitrarily among different device modes, just as normal users operate their smartphones. Classification of motion and device modes is a less studied area in literature, where we here mention a few studies. The classifier introduced in [13] is based on accelerations and magnetic field data 
recorded with a hand-held unit. Another study dealing with different motion models and device modes is performed and reported in [14], where standing still and walking patterns are studied. An extended investigation is to add also the running mode as in [12]. The classification of motion mode is also studied in [15]-[18].

The importance of the modes classification for PNS can be summarized as follows. The main design parameters include the step length and the step detection threshold determining when the magnitude of the measured acceleration is deemed to be caused by a step. Both these depend on the motion mode. Basically, the smaller step length, the smaller threshold is required. The device mode can simplify the model further. For instance, if the device is hand held flat, the heading corresponding to the projection (rotation) to the horizontal plane (heading) can be computed by just integrating the angular rate around the gravity vector. There are many other similar tricks described in literature. There is one recent proposal of a multi-mode PDR algorithm [17], otherwise mode-switching algorithms seem to be rare in literature.

The rest of the paper is organized as follows: Sec. II explains experimental setup in details, followed by description of available data in Sec. III. In Sec. IV data analysis and initial classification results, are presented. Finally, Sec. V concludes the work.

\section{EXPERIMENT SETUP}

In this section, we first introduce the hardware used in the experiments, then all measurement scenarios are described in detail. Finally, characteristics of all participants are presented to give a better comparison on signal behavior for different subjects with different attributes.

\section{A. Sensors}

The hardware can be grouped into two categories; high and low quality; MVN and Nexus 5, respectively. Subsequently, the signals from the mobile phones will be compared with the MVN system to have a better classification for mobile signals.

1) Xsens MVN Motion Capture: The Xsens MVN system has been used to capture the whole body motion. In the experiments the "MVN Awinda" system has been used. It contains 17 wireless Motion Trackers (MTw), an Awinda station, and MTw full body velcro straps.

The MTw is a miniature inertial measurement unit containing a 3D linear accelerometer, a 3D rate gyroscope, a 3D magnetometer, and a barometer.

The 17 trackers are placed at strategic locations on the body (secured by the straps), to measure motion of each body segment. Fig. 1 shows the location of the straps and attached MTws. The MVN system is controlled by the MVN studio software. A snapshot of the MVN system is shown In Fig. 2.

2) Nexus 5: Four Nexus 5 smartphones are carried by the subjects in the experiments. The Nexus 5 is equipped with multiple sensors; The sensors that are considered in this work are the A-GPS, the 3D linear accelerometer, the 3D rate gyroscope, and the barometer.

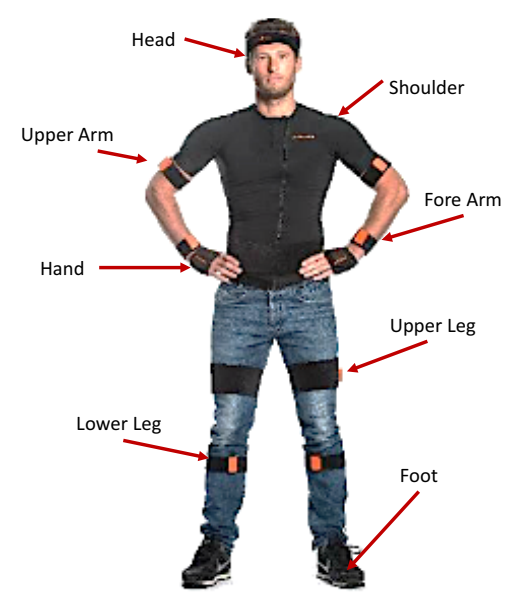

Fig. 1: MVN Awinda Straps [19].

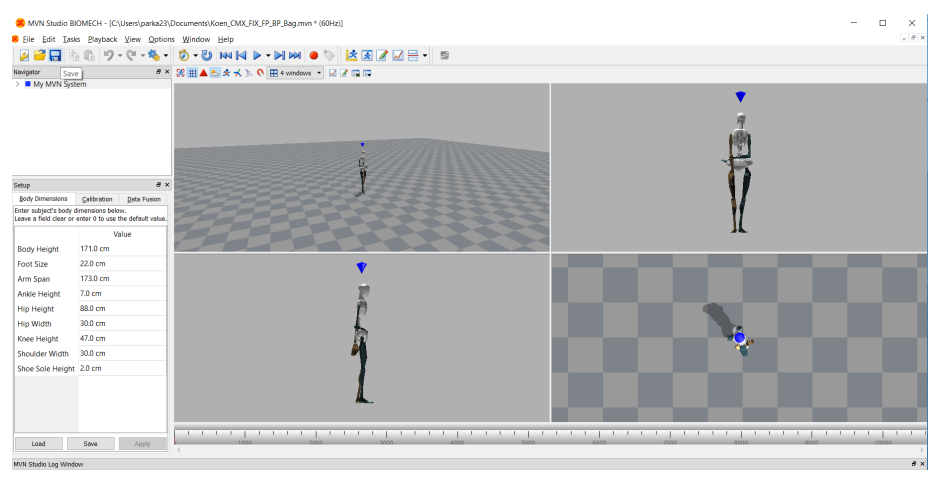

Fig. 2: MVN Studio BIOMECH application

The sensor fusion Android app [20], [21], installed on the Nexus 5 phones, is used to log the sensors measurements from the phones.

All phone locations used in the experiment are presented in Table I. A summary of the details about both the MVN system and the phone is provided in Table II; applications, sampling frequency, sensor's positions, and IMU sensors.

TABLE II: Measurement device specifications.

\begin{tabular}{|c|c|c|c|c|}
\hline Device & Application & $\begin{array}{c}\text { Sampeling } \\
\text { Frequency } \\
{[\mathrm{Hz}]}\end{array}$ & Position & Sensors \\
\hline Xsens & $\begin{array}{c}\text { MVN } \\
\text { Awinda }\end{array}$ & 60 & $\begin{array}{c}\text { Head } \\
\text { Shoulders } \\
\text { Upper Arms } \\
\text { Fore Arms } \\
\text { Upper Legs } \\
\text { Lower Legs } \\
\text { Hands } \\
\text { Feet } \\
\text { Pelvis }\end{array}$ & $\begin{array}{l}\text { Accelerometer } \\
\text { Gyroscope } \\
\text { Magnetometer } \\
\text { Barometer }\end{array}$ \\
\hline Nexus 5 & $\begin{array}{l}\text { Sensor } \\
\text { Fusion } \\
\text { app }\end{array}$ & 100 & $\begin{array}{c}\text { Hand } \\
\text { Front Pocket } \\
\text { Back Pocket } \\
\text { Backpack }\end{array}$ & $\begin{array}{c}\text { GPS } \\
\text { Accelerometer } \\
\text { Gyroscope } \\
\text { Magnetometer } \\
\text { Barometer }\end{array}$ \\
\hline
\end{tabular}




\section{B. Scenarios}

Measurements were collected in a building at Twente university. During the experiments the subjects walked three different paths, with a mixture of different motion modes, as represented in Table I. We followed certain rules when gathering the data so that the obtained measurements should mimic reality. For instance, to avoid any abnormal behavior the subjects were asked to carry one smartphone in the hand at each time. One run-through of the scenarios including a preparation phase lasts around 45-60 min.

Fig. 3 illustrates three different paths on top of the map of the area where measurements were obtained. The paths on the maps are for illustrative purposes and are inexact. For each path in Fig. 3, the subject holds one mobile phone in the hand, two more phones in front and back pockets, and one in the backpack. In this section, we thoroughly present the three measurement scenarios studied in this work. One scenario contains outdoor-only measurements, while the other two scenarios combine both outdoor and indoor paths. All three scenarios share the property that the measurement begins and ends at the same point, in an outdoor environment.

1) Outdoor-only, Standing Still, Walking and Running: The simplest scenario corresponds to Fig. 3a where the whole measurement is performed outdoors where the GPS signal is available. In this scenario, measurements are performed while the subject covers several motion and device modes, corresponding to Case 1 in Table III.

2) Outdoor-indoor, Walking: As in the previous scenario, the measurement starts and ends at the same point outside the building. However, a bit in to the track, the subject gets into the building and walks across a corridor, as illustrated in Fig. 3b. In this scenario, measurements are performed while the subject walks the whole path and holds the smartphon flat and fixed in the hand, corresponding to Case 2 in Table III.

3) Outdoor-indoor, Standing Still, Walking and Running: In this scenario, we consider the most complex behavior in terms of motion modes and also the experiment path. Along the path depicted in Fig. 3c, the subject starts outside the building, then gets into the building and passes one corridor, takes the stairs up and passes a certain distance, followed by taking the stairs down and getting back to the starting point. The designed path in this scenario, is measured for two different cases:

- Case 3: The subject walks along the path for both sets of device modes presented in Table III (each set is performed separately).

- Case 4: The subject has several motion modes along the path for both sets of device modes presented in Table III (each set is performed separately).

\section{Participants}

The described experiments were performed by twelve volunteers, 7 males and 5 females, with ages ranging from 25 to 45 years old. Due to some technical and practical issues (GPS signal loss outdoors, physical difficulties making it impossible for subjects to perform all the experiments), only 6 men and 2 women performed all the explained scenarios.
TABLE III: Measurement Scenarios. Device and motion modes are presented in Table I. Paths corresponding to each case are depicted in Fig. 3

\begin{tabular}{|c|c|c|c|c|}
\hline Scenario & $\begin{array}{c}\text { Device } \\
\text { Mode }\end{array}$ & $\begin{array}{c}\text { Motion } \\
\text { Mode }\end{array}$ & Participants & $\begin{array}{c}\text { Duration } \\
\text { (average) } \\
\text { [s] }\end{array}$ \\
\hline Case 1 & $1,2,3,4$ & W,R,SS & $\begin{array}{c}5 \text { Males } \\
\text { 2 Females }\end{array}$ & 190 \\
\hline Case 2 & $1,3,4$ & $\mathrm{~W}$ & $\begin{array}{c}\text { 6 Males } \\
\text { 2 Females }\end{array}$ & 200 \\
\hline Case 3 & $\begin{array}{c}1,3,4 ; \\
2,3,4\end{array}$ & $\begin{array}{c}\mathrm{W} \\
\text { Upstairs } \\
\text { Downstairs }\end{array}$ & $\begin{array}{c}\text { 6 Males } \\
\text { 2 Female }\end{array}$ & 280 \\
\hline Case 4 & $\begin{array}{c}1,3,4 ; \\
2,3,4\end{array}$ & $\begin{array}{c}\text { W,R,SS } \\
\text { Upstairs } \\
\text { Downstairs }\end{array}$ & $\begin{array}{c}\text { 6 Males } \\
\text { 2 Female }\end{array}$ & 270 \\
\hline
\end{tabular}

\section{AVAilable DATA}

This section provides a detailed explanation of how the data was collected followed by a description of the ground truth. Finally, we clarify the data structure as well as provide instructions on accessing different parts of the available data summarized in Table III.

\section{A. Collected Data}

Data is gathered from the scenarios in Sec. II-B using the hardware specified in Sec. II-A. Before each experiment/data collection, the MVN Awinda is calibrated. The calibration can be done by MVN studio in a less disturbed magnetic field area while the subject is standing in a fixed pose for around 5 seconds. Table III summarizes all the different scenarios. It is worth noting that the laptop connected to the Awinda station must be near the subjects while recording measurements due to short signal range. Fig. 4 shows a subject while doing an experiment. She is wearing Xsens suit and carrying several phones.

The available data for both smartphone and Xsens suits together with units of each measurement are presented in Table IV. Both the raw sensory data as well as the virtual IMU measurements from the Biomechanical (BM) model are extracted from MVN studio and provided in the dataset. Although MTws are equipped with a barometer, the MVN studio cannot extract this feature, therefore, the MTw data for the barometer is not given.

The phone data is only available in raw version. The GPS signal from the phones is converted to East-North-Up (ENU) coordinates, with the first measured GPS position considered as the reference point.

\section{B. Ground Truth}

The ground truth plays an important role in the classification process. The classifier uses part of the data for which the corresponding class is known as training data in order to establish the discriminating criteria. The part of the ground truth which was not used for training purposes will afterwards be used for the assessment of the classification accuracy. The remaining data with unknown class could then be assigned to a class. 


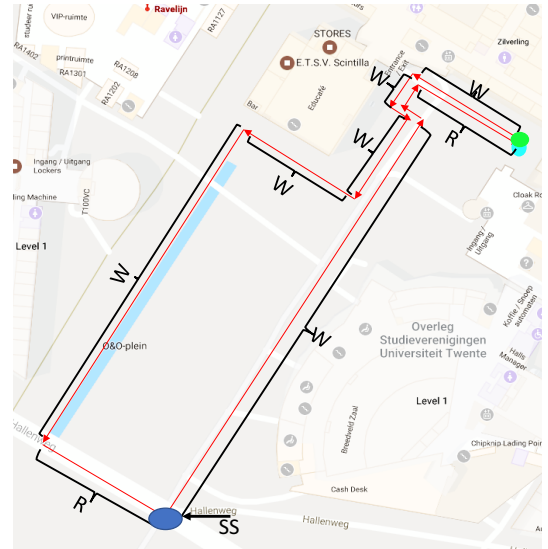

(a) Outdoor-only scenario with all classes (Case 1).

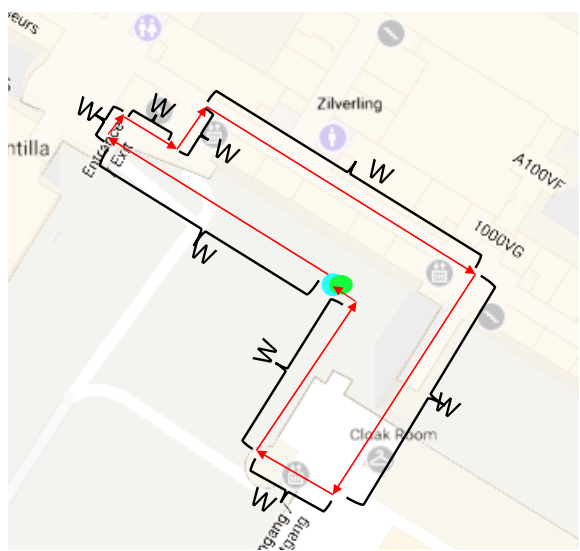

(b) Outdoor-Indoor scenario containing the W1 class (Case 2).

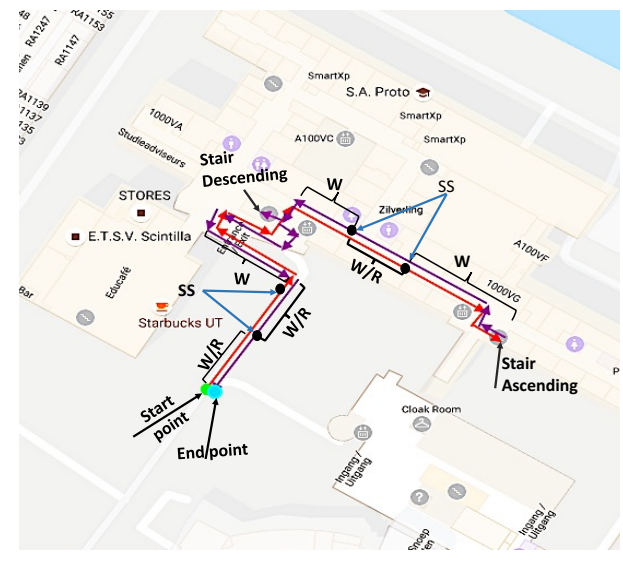

(c) Outdoor-Indoor scenario containing all classes. Forward and backward paths are indicated with red and purple colors, respectively (Case 3 and Case 4).

Fig. 3: Different measurement scenarios. The start and end points are indicated by green and cyan dots, respectively. All the motion modes and classes are defined based on Table I.

TABLE IV: Dataset details given from both smartphone and MTws. Signals from MTws are available in two versions; "Raw" data and filtered by some Biomechanical algorithms (BM). Smartphone data is only available in "Raw" version.

\begin{tabular}{|c|c|c|c|}
\hline Variable Names & Unit & MTw data & Phone data \\
\hline 3D AccR (Raw) & {$\left[\mathrm{m} / \mathrm{s}^{2}\right]$} & $*$ & $*$ \\
\hline 3D AngVelR(Raw) & {$[\mathrm{rad} / \mathrm{s}]$} & $*$ & $*$ \\
\hline 3D OriR (Raw) & {$\left[{ }^{\circ}\right]$} & $*$ & $*$ \\
\hline 3D MagR (Raw) & {$[\mathrm{Gauss}]$} & $*$ & $*$ \\
\hline GPS & {$[\mathrm{m}]$} & - & $*$ \\
\hline Pressure & {$[\mathrm{Pa}]$} & - & $*$ \\
\hline Mtw Position & {$[\mathrm{cm}]$} & $*$ & - \\
\hline Velocity & {$[\mathrm{m} / \mathrm{s}]$} & $*$ & - \\
\hline 3D Acc (BM) & {$\left[\mathrm{m} / \mathrm{s}^{2}\right]$} & $*$ & - \\
\hline 3D AngVel(BM) & {$[\mathrm{rad} / \mathrm{s}]$} & $*$ & - \\
\hline 3D Ang.Acc (BM) & {$\left[\mathrm{rad} / \mathrm{s}^{2}\right]$} & $*$ & - \\
\hline Ori (BM) & {$\left[{ }^{\circ}\right]$} & $*$ & - \\
\hline Joint Angle & {$\left[{ }^{\circ}\right]$} & $*$ & - \\
\hline Ground Truth (GT) & - & $*$ & $*$ \\
\hline
\end{tabular}

The classification accuracy refers to the correspondence between the class label assigned to each sample and the "true" class obtain by the ground truth. Fuzzy class boundaries and incorrectly assigned classes are two main degrading factors of classification accuracy. Additionally, if the ground truth does not represent all classes adequately the classification result and the corresponding accuracy may be unpredictable.

As Fig. 2 shows, MVN studio provides a movie of all the subject activities. We visually inspect these movies to determine the time of switch between the modes. However, the provided output of the MVN studio is a downsampled version of the signal. Thus, the visually obtained times need to be matched by the samples of the signal. We use the extracted output of the MVN studio that gives the exact time of each sample with miliseconds accuracy. All the samples are then labeled to appropriate classes using the derived time stamps used to form the ground truth.

The ground truth is separately formed for high and low quality devices using the labels obtained. Depending on the device mode, signals obtained from appropriate MTws should be considered. For example, if the goal is to investigate the scenario in which the smartphone is being carried in hand, the hand-mounted sensor is the one that mimics that behaviors best. Another example is the case where the phone is in pocket where the most appropriate MTw revealing same properties is the one mounted on the upper leg. Similarly, we further assumed the MTw on the pelvis to simulate the scenario where the phone is being carried in backpack.

\section{Acquire Data}

The dataset containing the scenarios defined in Table III and is available from [22]. The logged data from the phones and the MVN studio are extracted with 100 and $60 \mathrm{~Hz}$ sampling frequencies, respectively. Data from both devices are merged into a MATLAB '.mat'-file. The naming of experiments is in line with the structure given in Table III. This file contains the structure of the data and attributes corresponding to each subject. For example, all measurements related to Case 4, are stored in Case 4 dataset and Case 4 . Subject (1) provides the data for all sensors and attributes associated with subject 1 .

Table IV represents all available data for both devices in the measurement scenarios dataset. During the post-analysis phase of the data, some signals were identified as either corrupted or missing. As a result, the dataset contains some empty fields. The dataset contains 4 measurement scenarios with 7-8 measurement sets each, depending on the number of participants. To further simplify working with the dataset, a toy example with MATLAB code to extract the data is provided together with the dataset. The first measurement scenario (Case 1) for first subject is set as default. More details about setting variables and extracting desired outputs is provided in a README file attached to the dataset. 


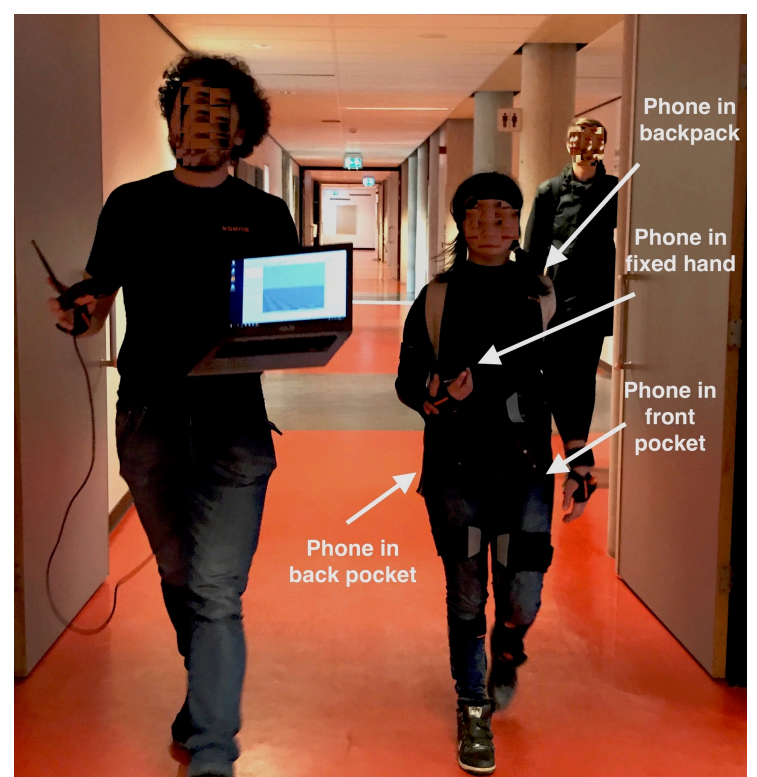

(a) The subject is walking constantly with almost constant speed and carrying a smartphone in flat and fixed hand.

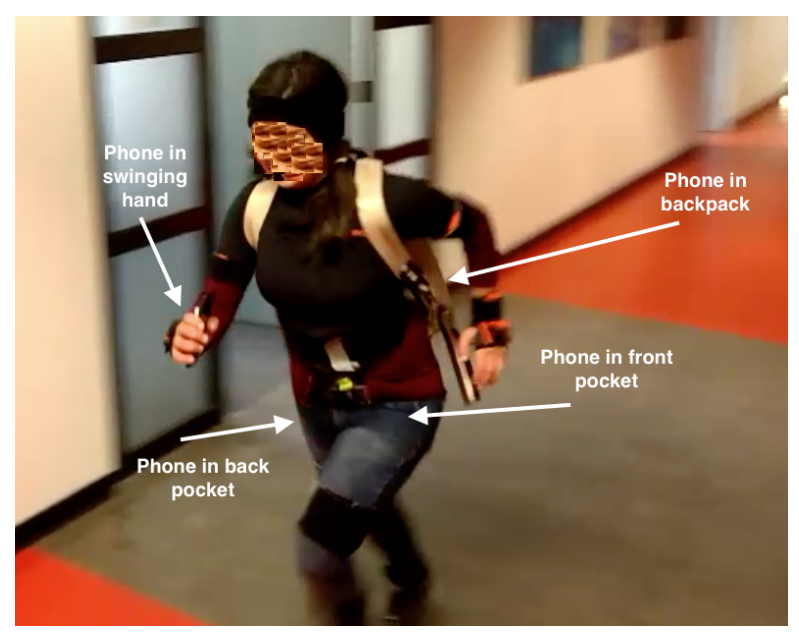

(b) The subject is running and carrying phone in swinging hand.

Fig. 4: photo from measurement campaign.

\section{DATA ANALYSis}

We apply a classification algorithm to the data to assign them to the defined classes in Table I. This is done in two steps; feature extraction on the raw signals, followed by a classification step.

\section{A. Feature Extraction}

Feature extraction is a way to try to better bring out the inherent information in the available data, and reduce the dimensionality of the raw/pre-processed data in order to be able to apply classification algorithms on it.

The feature extraction phase is performed by dividing the inertial data in sliding windows of $N$ samples with no overlap. The window size must be selected such that it satisfies two different objectives. On one hand, it must be long enough to cover at least one gait cycle. On the other hand, it should be short enough to identify sudden motion mode transitions.

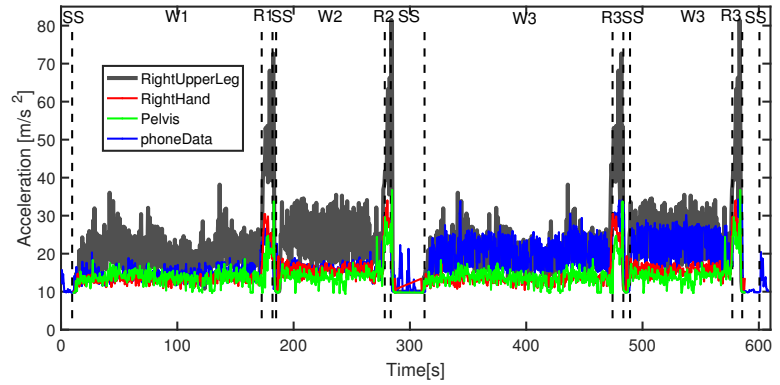

(a) Accelerometer norm of signal.

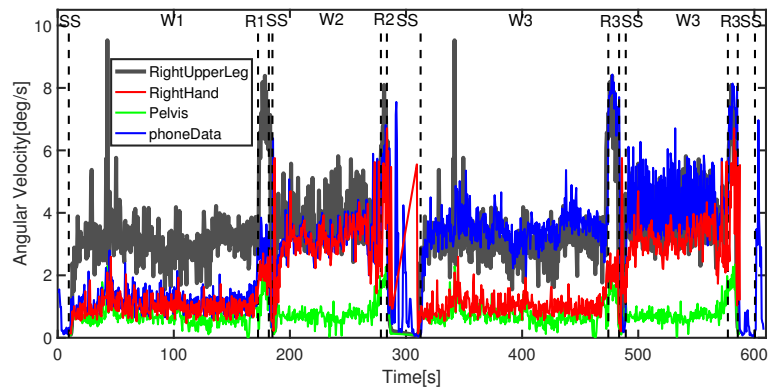

(b) Gyroscope norm of signal.

Fig. 5: Signal norm for 4 different motion trackers together with the signal from the smartphones. The subject performed the Case 1 from Fig. 3. Black lines separate the different classes. Related class for each section is indicated in the figures. The subject carried a smartphone in the right hand and one in the front right pocket. The device carried by hand is switching between fixed and swinging mode.

In this work, we set the window size to 0.5 seconds. This translates to 50 and 30 samples for phone and motion trackers, respectively, imposed by their sampling frequencies.

In the rest of this section we define the features we feed to the classifier and provide more specific examples from the dataset. All given examples correspond to Case 1 described in Table III where the subject intentionally switches between the fixed and swinging device modes.

1) Signal Norm: For a generic signal $S[n],\|S\|_{\max }$ denotes the maximum norm over the sampling window as follow,

$$
\|S\|_{\max }=\max _{n}\|S[n]\| \text {. }
$$

The norm contains useful information used discriminate between different modes. More precisely, any change of motion mode results in a large difference in the values of accelerometer norm, $\|a\|_{\max }$. This enables the identification of a change in the motion mode. In addition, large difference in the values of the gyroscope norm $\|\omega\|_{\max }$ can be translated into a switch in the device mode.

To further illustrate the behavior of $\|a\|_{\max }$ relative to the change in the motion mode see Fig. 5, where the norms of signals are depicted while the smartphone is in both fixed and swinging modes. These signals correspond to the Case 1 in Fig. 3. Fig. 5b further presents how the gyroscope norm, $\|\omega\|_{\max }$, assists in device mode discrimination. 


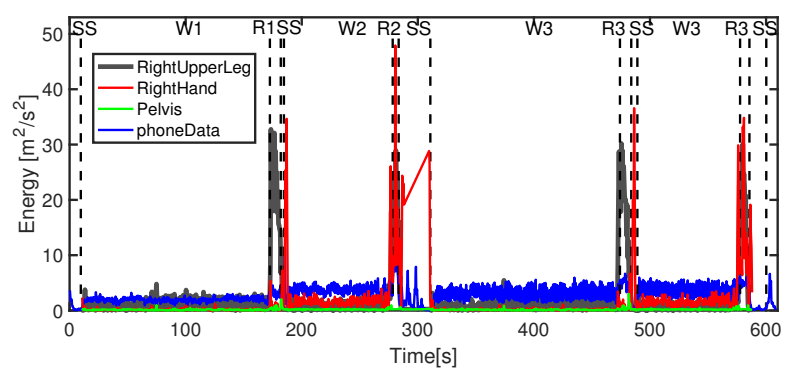

(a) Accelerometer energy signal.

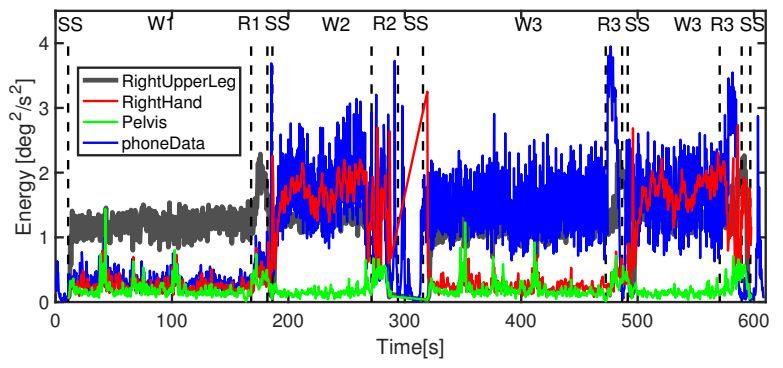

(b) Gyroscope energy signal.

Fig. 6: Energy signal for 4 different motion trackers together with the signal from the smartphones. The sensor locations and measurement scenario are the same as the one described in Fig. 5.

2) Signal Energy: Let $S[n]$ be a generic signal, e.g. a single accelerometer/gyroscope direction or accelerometer/gyroscope norm. The energy of the signal $E_{S}$ is obtained by taking the squared norm of $S[n]$ and summing and normalizing it over the sliding window:

$$
E_{S}=\frac{1}{N} \sum_{n=0}^{N-1} S[n]^{2}
$$

The obtained energy is a useful feature allowing us to distinguish fixed or moving device modes due to rapid response to mode transition. For example, the gyroscope will have higher energies in swinging hand device mode than the fixed hand scenario.

Fig. 6 shows the energy signal for both accelerometer and gyroscope. As shown in Fig. 6a each transition between motion modes, results in a noticeable change in the energy of the accelerometer signal. The effect of the device mode on the energy signal is illustrated in Fig. $6 \mathrm{~b}$, where it is shown that switching from fixed to swinging mode increases the angular velocity energies drastically.

3) Signal variance: For any generic signal $S[n]$ the average of the squared differences from the mean, the variance signal, is defined as follows:

$$
\sigma_{S}^{2}=\frac{1}{N-1} \sum_{n=0}^{N-1}\left(\|S[n]\|-\frac{1}{N} \sum_{n=0}^{N-1}\|S[n]\|\right)^{2} .
$$

The variance signal assists to discriminate between high and low intensity movements. For example, the estimated variance of both accelerometer and gyroscope is highly informative

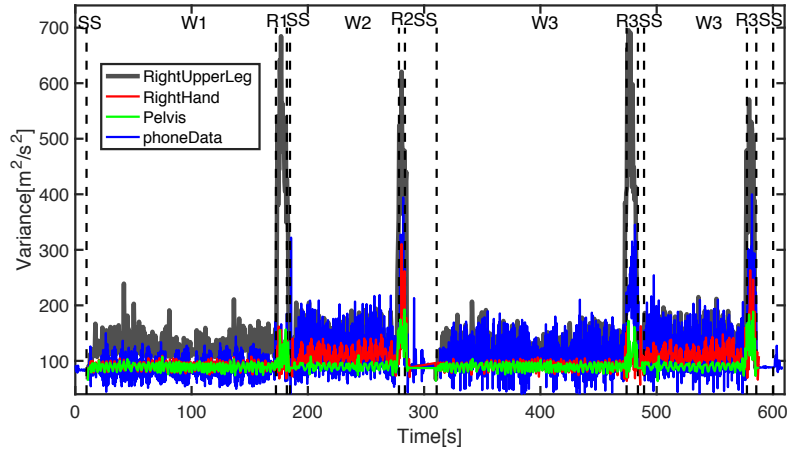

(a) Accelerometer signal variance.

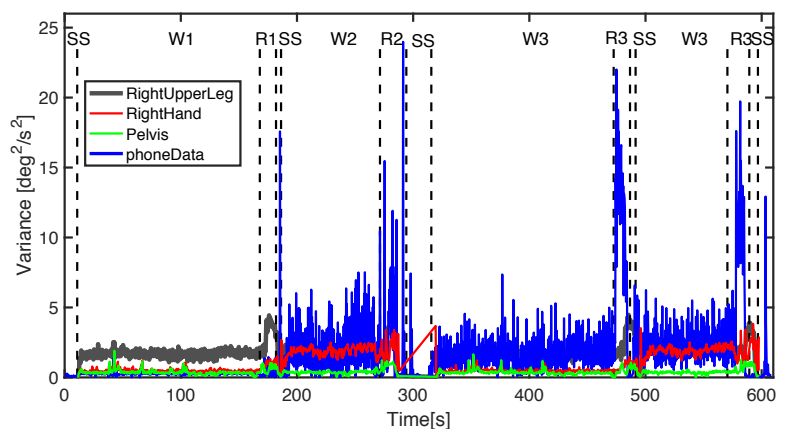

(b) Gyroscope signal variance.

Fig. 7: Signal variance for 4 different motion trackers together with the signal from the smartphones. The sensor locations and measurement scenario are the same as the one described in Fig. 5.

while the objective is to distinguish between swinging mode with any other less intense movement scenarios.

Fig. 7 shows the signal variance of both accelerometer and gyroscope. One advantage of the variance signal is to enhance robustness of the classification algorithm in motion mode recognition. Running leads to more intense movements than both walking and standing still. Higher peaks in the signal variance, as shown in Fig. 7a, can then be identified once the user switches to this mode. Additionally, the signal variance analysis can be used to recognize device mode switches. The variation of the variance signal illustrated in Fig. 7b shows how changing between classes with different device modes, W1 and W2 for example, can be distinguished.

4) Frequency Analysis: This feature allows us to identify any movement such as periodic movement from aperiodic ones. Different activities have different frequencies, resulting in a varying power spectrum that assists in activity identification. Thus, the analysis of the frequency domain of inertial signals recorded with hand-held devices allows capturing the periodicity of the accelerometer/gyroscope signals due to the subject's activity. Presence or absence of peaks in the spectogram of the inertial signals gives useful insights whether the subject is having a periodic movement or standing still.

The spectogram of the gyroscope signal is obtained using Short Time Fourier Transform (STFT) and reported in Fig. 8. The periodicity of the walking and running is visible in the 
frequency peaks of the spectogram while it is possible to identify a static case around time 300 .

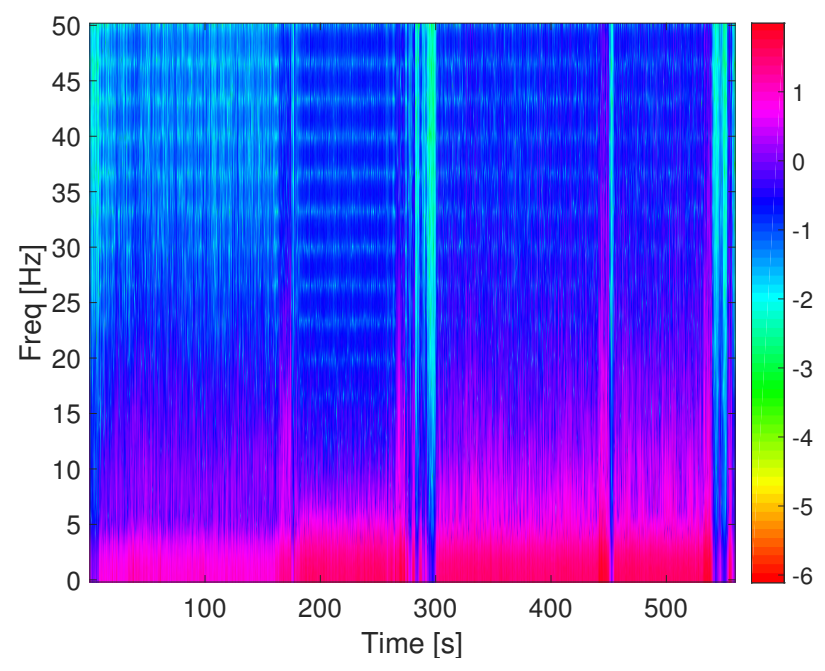

Fig. 8: Spectrogram of the gyroscope signal for the right hand from a smartphone. The subject performed the Case 1 from Fig. 3.

\section{B. Classification}

As a final step in characterizing the data, a classifier is applied to the features to try extract the motion and device modes as defined in Table I.

Different machine learning algorithms can be used for the classification. For example, [14] applies a decision tree for classification purposes while [12] uses multi-layer perceptron (MLP) and a support vector machine (SVM) to improve the performance in terms of recognition of human activity.

Two datasets, are formed for phone and motion tracker signals as described in Sec. III-B, separately. Each set contains eight features constructed from four signal attributes introduced in Sec. IV for both gyroscope and accelerometer. Since the classes are labeled accurately for each dataset, a supervised learning approach is applied. A multivariate decision tree classifier is trained using MATLAB; Machine learning toolbox. The classifier is validated with 10 -fold crossvalidation.

The performance of the classifier for both devices are summarized in the confusion matrices given in Tables Va and $\mathrm{Vb}$. In the MTw data, having a sensor to mimic the behavior of the phone data carried by backpack is challenging. As Table Va reports, the mode detection success rate for MTw sensors are over $70 \%$ for all classes. SS and $\mathrm{W} 1$ classes are missclassified with W4 class corresponding to the pelvis sensor. To explain this observation, one needs to note how the pelvis sensor is rigidly mounted on the body. This leads to miss-classification with other low intensity modes. With phone data, the classification results are promising as represented by Table Vb. All the classes are detected correctly with over $75 \%$ for walking and standing still modes and over $56 \%$ for running mode. Running mode recognition is challenging as the phone has movement. Missclassification of R1 with R4 and W1 with W4 can be explained by the fact that the phone is fixed in either of these situations.

\section{CONCLUSION}

This paper presents an extensive dataset for classification and investigation of the motion and device mode for pedestrian navigation systems (PNS), where many device modes, a number of users and a few motion modes are logged for three different trajectories. All data are fully annotated with ground truth classes, and other metadata such as position and accurate full body motion using biomeachnical models. We have discussed how the motion and device mode relate to pedestrain dead-reckoning algorithms, the working horse of PNS, and how PNS can otherwise benefit from improved and extended mode classification. The larger part of the paper described the field tests in detail. The last part suggests a few features that can be computed from the logged data, and applies a straight forward classifier on these features. The result is quite promising, yet preliminary. The dataset is publicly available [22], and we hope the research community can benefit from this to improve the classification results further.

\section{ACKNOWLEDGMENT}

The authors would like to thank Dr. P. K. Mandal, PhD students, and secretaries from mathematics and computer science department, Twente University who voluntarily participated to the data collection experiment. Additionally, we would like to thank Xsens for providing the measurement equipment.

This work is funded by the European Union FP7 Marie Curie training program on Tracking in Complex Sensor Systems (TRAX) with grant number 607400.

\section{REFERENCES}

[1] P. Kasebzadeh, C. Fritsche, G. Hendeby, F. Gunnarsson, and F. Gustafsson, "Improved pedestrian dead reckoning positioning with gait parameter learning," in International Conference on Information Fusion, Heidelberg, Germany, Jul. 2016.

[2] E. Foxlin, "Pedestrian tracking with shoe-mounted inertial sensors." IEEE Comput. Graph., vol. 25, p. 38-46, 2005.

[3] S. Beauregard, "Omnidirectional pedestrian navigation for first responders," in 4th Workshop on Positioning, Navigation and Communication (WPNC), Hannover, Germany, Mar. 2007.

[4] H. J. Luinge and P. Veltink, "Inclination measurement of human movement using a 3-D accelerometer with autocalibration," IEEE Transactions Neural System Rehabil. Eng., vol. 12, no. 1, pp. 112-121, Mar. 2004.

[5] J. C. Alvarez, D. Alvarez, A. López, and R. C. González, "Pedestrian navigation based on a waist-worn inertial sensor," Sensors, vol. 12, no. 8, pp. 10 536-10 549, 2012.

[6] J. Li, Q. Wang, X. Liu, and M. Zhang, "An autonomous waistmounted pedestrian dead reckoning system by coupling low-cost MEMS inertial sensors and GPS receiver for 3D urban navigation," Journal of Engineering Science and Technology, vol. 7, no. 9, pp. 9-14, 2014.

[7] U. Steinhoff and B. Schiele, "Dead reckoning from the pocket-an experimental study," in IEEE International Conference on Pervasive Computing and Communications (PerCom), Mannheim, Germany, Mar. 2010.

[8] C. Gusenbauer, D. Isert and J. Krosche, "Self-contained indoor positioning on off-the-shelf mobile devices." in Proceedings of IEEE 2nd Conference on Indoor Positioning and Indoor Navigation, Zurich, Switzerland, Sep. 2010. 
TABLE V: Confusion matrix of the modes classification represented in Table I using the binary decision tree classifier using cross-validation with 10 folds. The table shows how different annotated activities are classified in [\%].

(a) Data from 4 different motion trackers

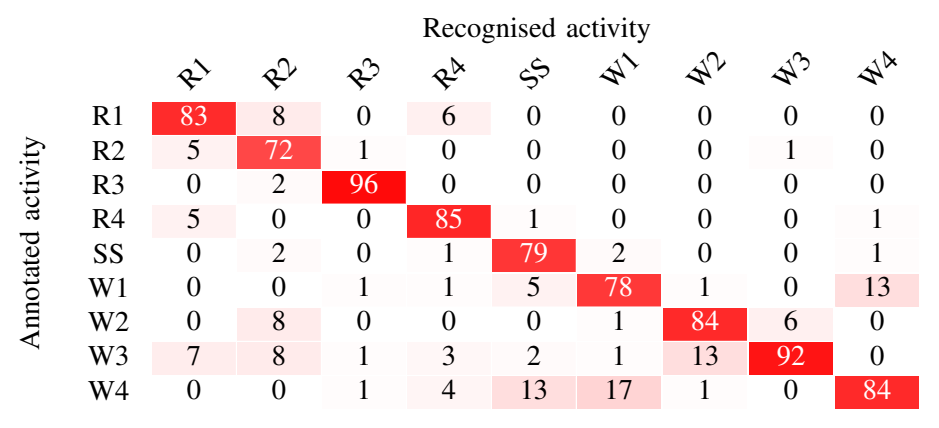

(b) Data from phones.

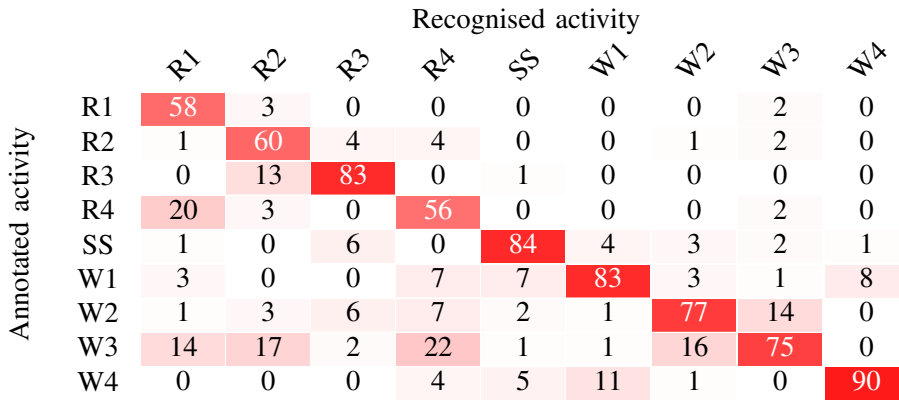

[9] Y. Liu, Y. Chen, L. Shi, Z. Tian, M. Zhou, and L. Li, "Accelerometer based joint step detection and adaptive step length estimation algorithm using handheld devices," Journal of Communications, vol. 10, no. 7, pp. 520-525, 2015.

[10] Z. Tian, Y. Zhang, M. Zhou, and Y. Liu, "Pedestrian dead reckoning for MARG navigation using a smartphone," EURASIP Journal on Advances in Signal Processing, vol. 16, no. 1, pp. 2-11, 2014.

[11] S. Tomažič and I. Škrjanc, "Fusion of visual odometry and inertial navigation system on a smartphone," Elsevier on Computers in Industry, vol. 74, pp. 119-134, 2015.

[12] H. Zhang, W. Yuan, Q. Shen, T. Li, and H. Chang, "A handheld inertial pedestrian navigation system with accurate step modes and device poses recognition,” IEEE Sensors Journal, vol. 15, no. 3, pp. 1421-1429, 2015.

[13] L. Pei, J. Liu, R. Guinness, Y. Chen, H. Kuusniemi, and R. Chen, "Using LS-SVM based motion recognition for smartphone indoor wireless positioning." Sensors, vol. 12, p. 6155-6175, 2012.

[14] V. Susi, M. Renaudin and G. Lachapelle, "Motion mode recognition and step detection algorithms for mobile phone users," Sensors, vol. 13, no. 2, p. 1539-1562, 2013.

[15] M. Elhoushi, J. Georgy, A. Noureldin, and M. Korenberg, "Motion mode recognition for indoor pedestrian navigation using portable devices," IEEE Transactions on Instrumentation and Measurement, vol. 65, no. 1, pp. 208-221, 2016.

[16] 1 , "A survey on approaches of motion mode recognition using sensors," IEEE Transactions on Intelligent Transportation Systems, vol. PP, no. 99, pp. 1-25, 2016.

[17] Q. Tian, K.-K. Salcic, Z. Wang, and Y. Pan, "A multi-mode dead reckoning system for pedestrian tracking using smartphones," IEEE Sensors Journal, vol. 16, no. 7, pp. 2079 - 2093, 2016.

[18] A. Reiss, G. Hendeby, and D. Stricker, "A competitive approach for human activity recognition on smartphones," in European Symposium on Artificial Neural Networks, Computational Intelligence and Machine Learning (ESANN), Bruges, Belgium, Apr. 2013.

[19] MVN Users Manual. User Guide MVN, MVN BIOMECH, MVN Link, MVN Awinda, Xsens, Nov. 2016. [Online]. Available: https://issuu.com/xsensmvn/docs/mvn_user_manual_ $71 \mathrm{c} 37181653 \mathrm{db} 5 ? \mathrm{e}=14522406 / 12478179$

[20] Linköping University, Sweden. (2014, Dec.) Sensor fusion app. [Online]. Available: https://goo.gl/0qNyU

[21] G. Hendeby, F. Gustafsson, N. Wahlström, and S. Gunnarsson, "Platform for teaching sensor fusion using a smartphone," International journal of engineering education, vol. 33, no. 2(B), pp. 781-789, 2017.

[22] IMU Dataset for Device and Motion Mode Classification. [Online]. Available: http://users.isy.liu.se/rt/parka23/research.html 